The ASTRONOMICAL JouRNAL, 120:1963-1973, 2000 October

(2000. The American Astronomical Society. All rights reserved. Printed in U.S.A.

\title{
A SEARCH FOR INTERSTELLAR BUBBLES SURROUNDING MASSIVE STARS IN PERSEUS OB1
}

\author{
C. E. CAPPA ${ }^{1}$ \\ Instituto Argentino de Radioastronomía, CC 5, 1894 Villa Elisa, Argentina; and Facultad de Ciencias Astronómicas y Geofísicas, Universidad Nacional de \\ La Plata, Paseo del Bosque s/n, 1900 La Plata, Argentina; ccappa@, fcaglp.fcaglp.unlp.edu.ar \\ AND \\ UWE HERBSTMEIER \\ Max-Planck-Institut für Astronomie, Königstuhl 17, D-69117 Heidelberg, Germany; and Radioastronomisches Institut der Universität Bonn, Auf dem \\ Hügel 71, D-53121 Bonn, Germany \\ Received 2000 March 31; accepted 2000 June 21
}

\begin{abstract}
We have examined the interstellar medium in the vicinity of massive stars belonging to the Per OB1 association based on neutral hydrogen $21 \mathrm{~cm}$ observations obtained with the $100 \mathrm{~m}$ radio telescope at Effelsberg (HPBW = 8.4) and complementary data from the Leiden-Dwingeloo H I Survey $\left(\mathrm{HPBW}=36^{\prime}\right)$. The higher angular resolution $\mathrm{H}$ I observations allowed us to discover probable windblown bubbles related to four massive stars in the association, namely, HD 14442 [O5n(f)p], HD 14947 [O5If +$]$, HD 13022 [O9.5II-III((n))], and HD 13338 [O9.5V], while the detection of a wind-blown bubble associated with HD 16691 [O5If + ] is less conclusive. A clear H I shell coincident in position with two B1III stars (HD 15233 and Hilt 311) was also detected. Some of these features also have infrared and/or molecular counterparts. The energetics of the structures related to each massive star is analyzed. The new H I interstellar bubbles appear to be similar to the ones found surrounding WolfRayet stars and other Of stars. The large-scale maps obtained using the lower angular resolution H I data show that most of the early-type stars belonging to Per OB1 are placed in a region of low H I emission. The association could have blown a $\mathrm{H}$ I shell of about $350 \times 550 \mathrm{pc}$ in size. This large $\mathrm{H} \mathrm{I}$ shell has an infrared counterpart.
\end{abstract}

Key words: ISM: bubbles - ISM: individual (Perseus OB1) — stars: early-type — stars: individual

\section{INTRODUCTION}

H I $21 \mathrm{~cm}$ line observations have shown neutral hydrogen bubbles created by a hypersonic stellar wind interacting with the surrounding interstellar medium (ISM). These interstellar wind-blown bubbles (WBBs) appear associated with a large number of Wolf-Rayet (WR) stars (e.g., Marston 1996; Arnal et al. 1999; Cappa et al. 1999, and references therein). In most cases, their large dimensions together with their low expansion velocities suggest dynamical ages of a few million years, several times longer than the duration of the WR phase of a massive star $\left(\approx 5 \times 10^{5} \mathrm{yr}\right.$, Maeder \& Meynet 1994), implying that the massive O-type star progenitor of the current WR star must also be responsible for the formation of the bubbles.

Consequently, $\mathrm{O}$ and $\mathrm{Of}$ stars seem to be promising candidates for showing the signature of the interaction of the stellar wind with the surrounding medium. At optical wavelengths, Lozinskaya (1982) found ring nebulae associated with a few Of stars. Several neutral hydrogen bubbles were also detected surrounding Of stars (e.g., Kutchar \& Bania 1993; Cappa \& Benaglia 1998; Benaglia \& Cappa 1999). However, up to present, the number of well-studied associations of Of stars and $\mathrm{H}$ I voids and shells is too small for a comprehensive understanding of such interactions.

The analysis of the H I WBBs provides valuable information concerning the energetics and dynamics of the ISM. The comparison of these results with theoretical predictions from evolutionary models of interstellar bubbles allows us

\footnotetext{
${ }^{1}$ Member of Carrera del Investigador Científico, CONICET, Argentina.
}

to improve the modeling of the interaction between massive stars and the surrounding ISM.

The energy supplied to the ISM by massive stars in OB associations and open clusters also leads to the formation of shells and supershells through ionizing stellar radiation, stellar winds, and supernova explosions (e.g., Bruhweiler et al. 1980; McCray \& Kafatos 1987; Mac Low \& McCray 1988; Oey 1996). In particular, neutral hydrogen shells and supershells appear to be related to a certain number of galactic OB associations and open clusters (e.g., Per OB2, Sancisi 1974; Cep OB3, Simonson \& van Someren Greve 1976; Upper-Centaurus Lupus and Upper-Scorpius, de Geus 1988; Ori OB1b, Chromey, Elmegreen, \& Elmegreen 1989; Lac OB1, Cappa de Nicolau \& Olano 1990; Ara OB1, Rizzo \& Bajaja 1994; Car OB2, Rizzo 1994; and OCl 352, Normandeau, Taylor, \& Dewdney 1996).

In this paper we analyze the $\mathrm{H}$ I gas distribution in the environs of Per OB1 with the aim of investigating the action of the strong stellar winds of $\mathrm{O}$ and Of stars on their surroundings.

Per OB1 is an OB association spread within an area of $8^{\circ} \times 6^{\circ}$ approximately centered at $(l, b)=(134.5,-3.5)$. Humphreys (1978) derived a mean distance modulus of $11.83(2.3 \mathrm{kpc})$ for the association from the individual stellar distances of the O-, B-, and A-type stars, in agreement with previous results from Ruprecht (1966). Garmany \& Stencel (1992) obtained a distance modulus of 11.8 based on the best fit to a color-magnitude diagram for the B mainsequence stars. Distances to the open clusters $h$ and $\chi$ Persei [NGC 869 at $(l, b)=(134.5,-3.5)$ and NGC 884 at $(l, b)=$ $(135.1,-3.6)]$, which are generally considered to be related to Per OB1, range from 2.1 to $5.5 \mathrm{kpc}$ (Becker \& Fenckart 1971; Janes \& Adler 1982). However, Garmany \& Stencel 
cast some doubts on the fact that the clusters are related to the association. Based on these results, an optical distance of $2.3 \mathrm{kpc}$ will be adopted for Per OB1.

Heiles (1979) in his search for galactic shells and supershells, found an $\mathrm{H}$ I shell that correlates in position with Per OB1. This $5^{\circ}$ diameter structure is detected in the velocity range -31 to $-23 \mathrm{~km} \mathrm{~s}^{-1}$ (all velocities in this paper are referred to LSR). The presence of gas expanding away from Per OB1 is reinforced by studies of interstellar absorption lines in direction to several stars in the association (Münch 1957; Phillips \& Gondhalekar 1981; McLachlan \& Nandy 1985).

Koo, Heiles, \& Reach (1992) identified in this region the worm candidates GW 131.3-2.2 and GW 132.6-9, in the velocity ranges -59.6 to -16.9 and -31.3 to $-22.9 \mathrm{~km}$ $\mathrm{s}^{-1}$, respectively. These worms were also detected in infrared and radio continuum emission at 100 and $60 \mu \mathrm{m}$ and at 408 MHz. Heiles (1984) interpreted these structures as the walls surrounding superbubbles which have broken through the thin gaseous disk.

The stellar data of the $\mathrm{O}$ and $O$ f stars that are members of Per OB1 (Garmany \& Stencel 1992) are summarized in Table 1. Column (1) lists the name of the star; column (2), the galactic coordinates $(l, b)$; column $(3)$, its spectral classification; column (4), the stellar distance; columns (5) and (6), the stellar wind parameters; and column (7), the LSR stellar radial velocity. The stellar distances were estimated from photometric data (Nicolet 1978), and absolute magnitudes and intrinsic colors by Vacca, Garmany, \& Schull (1996) and Schmidt-Kaler (1982), respectively. A typical uncertainty of $25 \%$ has been taken into account for the distances. Within errors, the individual distances agree with the optical distance to Per OB1. Values in parenthesis in columns (5) and (6) indicate that $\dot{M}$ was derived through the fitting formula by Lamers \& Leitherer (1993) as a function of the luminosity and the effective temperature of the star, and $V_{w}$ corresponds to mean values obtained as a function of spectral type and luminosity class (Prinja, Barlow, \& Howarth 1990). The uncertainties in $\dot{M}$ and $V_{w}$ are $\simeq 0.2$ dex and $400 \mathrm{~km} \mathrm{~s}^{-1}$, respectively.

Our study is based on $\mathrm{H}_{\mathrm{I}} 21 \mathrm{~cm}$ line emission data obtained with the $100 \mathrm{~m}$ single-dish antenna of the MaxPlanck-Institut für Radioastronomie (MPIfR) at Effelsberg, Germany, with an angular resolution of 8.4, on low angular resolution observations taken from the $H$ I survey by Hartman \& Burton (1997), and on complementary infrared, radio continuum, and molecular data.

\section{DATA SETS}

We have performed $\lambda 21 \mathrm{~cm} \mathrm{H}$ I line observations over the region of the Per OB1 association where most of its $\mathrm{O}$ and Of stars are projected in 1992 January and 1993 December with the Effelsberg $100 \mathrm{~m}$ single-dish radio telescope of the MPIfR, Bonn, Germany. A region of $27.3 \mathrm{deg}^{2}$ was surveyed following two different grids. Two areas of $1 \mathrm{deg}^{2}$ each surrounding HD 14947 and HD 16691 were sampled with a grid spacing of $5^{\prime}$; while a grid spacing of $10^{\prime}$ was used for the rest of the region.

The dual-channel FET amplifier and the 1024 autocorrelation spectrometer, which was split in two halves with 512 channels each, were used to receive both independent circular polarizations. The frequency switching technique was applied with signal and reference bands $3.2 \mathrm{MHz}$ apart. Table 2 lists the main parameters of the observations. The brightness temperature scale was derived from observations of the calibration point position $\mathrm{S} 7\left(l=132^{\circ}, b=-1^{\circ}\right.$;

TABLE 1

O and Of Stars in Perseus Ob1: Stellar Data

\begin{tabular}{|c|c|c|c|c|c|c|}
\hline $\begin{array}{l}\text { Name } \\
\text { (1) }\end{array}$ & $\begin{array}{l}l, b \\
(2)\end{array}$ & $\begin{array}{l}\text { Sp. Class. } \\
\text { (3) }\end{array}$ & $\begin{array}{c}d \\
(\mathrm{kpc}) \\
(4)\end{array}$ & $\begin{aligned} & \dot{M} \\
\left(10^{-6}\right. & \left.M_{\odot} \mathrm{yr}^{-1}\right) \\
& (5)\end{aligned}$ & $\begin{array}{c}V_{w} \\
\left(\mathrm{~km} \mathrm{~s}^{-1}\right) \\
(6)\end{array}$ & $\begin{array}{c}\text { R.V. } \\
\left(\mathrm{km} \mathrm{s}^{-1}\right) \\
(7)\end{array}$ \\
\hline HDE 236894 . & $130.82,-3.48$ & $08 V^{a}$ & $3.1 \pm 0.8$ & $(0.3)$ & $(1800)$ & $-30^{\mathrm{b}}$ \\
\hline HILT $23 \ldots \ldots \ldots$ & $132.93,-1.38$ & O9V & $2.1 \pm 0.5$ & $(0.1)$ & $(1400)$ & \\
\hline HD $13022 \ldots \ldots \ldots$ & $132.90,-2.58$ & O9.5II-III $((\mathrm{n}))^{\mathrm{c}}$ & $2.8 \pm 0.7$ & $(0.4)$ & $(1500)$ & $-62^{d}$ \\
\hline HD $12323 \ldots \ldots \ldots$ & $132: 92,-5: 87$ & ON9V & $3.3 \pm 0.8$ & $0.004^{\mathrm{e}}$ & $1175^{f}$ & $-96^{\mathrm{g}},-51^{\mathrm{b}}$ \\
\hline HD $12993 \ldots \ldots \ldots$ & $133: 11,-3: 40$ & $06.5 \mathrm{~V}^{\mathrm{c}}$ & $2.9 \pm 0.7$ & $0.4^{\mathrm{h}}$ & $2335^{\mathrm{f}}$ & $\begin{array}{c}-141^{\mathrm{i}},-122^{\mathrm{g}} \\
-87^{\mathrm{b}}\end{array}$ \\
\hline HD 13338. & $133: 52,-3: 27$ & $\mathrm{O} 9.5 \mathrm{~V}^{\mathrm{d}}$ & $2.1 \pm 0.5$ & $(0.1)$ & $(1500)$ & \\
\hline HD $13268 \ldots \ldots \ldots$ & $133: 95,-5: 00$ & $\mathrm{ON}_{8} \mathrm{~V}^{\mathrm{c}}$ & $1.9 \pm 0.5$ & $0.7^{\mathrm{h}}$ & $2140^{f}$ & $\begin{array}{c}-132^{\mathrm{i}},-128^{\mathrm{b}} \\
-120^{\mathrm{g}}\end{array}$ \\
\hline HD 14442. & $134.20,-1.32$ & $\operatorname{O5n}(f) p^{c}$ & $2.9 \pm 0.7$ & (3.0) & $(2300)$ & $-50^{\mathrm{b}},-48^{\mathrm{g} . \mathrm{j}}$ \\
\hline HD $13745 \ldots \ldots \ldots$ & $134.58,-4.95$ & $09.7 \mathrm{II}((\mathrm{n}))^{\mathrm{c}}$ & $2.5 \pm 0.6$ & $0.5^{\mathrm{h}}$ & $1905^{f}$ & $-36^{\mathbf{i}},-27^{\mathrm{d}}$ \\
\hline HD $14947 \ldots \ldots \ldots$ & $134.98,-1.77$ & O5If $+^{c}$ & $2.6 \pm 0.6$ & $\begin{array}{c}3.0 \pm 0.7^{\mathrm{k}} \\
4.8 \pm 2^{1} \\
<18^{1} \\
7.5^{\mathrm{n}}\end{array}$ & $\begin{array}{l}1885^{\mathrm{f}} \\
2300^{\mathrm{m}} \\
2350^{\mathrm{n}}\end{array}$ & $\begin{array}{c}-86^{\mathrm{g}},-58^{\mathrm{i}} \\
-48^{\mathrm{j}}\end{array}$ \\
\hline HD $14434 .$. & $135: 07,-3: 82$ & $05.5 \mathrm{Vn}\left((\mathrm{f}) \mathrm{p}^{\mathrm{o}}\right.$ & $2.7 \pm 0.7$ & $\leq 0.7^{e}$ & $1960^{\mathrm{f}}$ & $\begin{array}{c}-33^{\mathrm{b}},-25^{\mathrm{i}} \\
-47^{\mathrm{g}}\end{array}$ \\
\hline HD 15642 . & $137.07,-4.73$ & O9.5III: $\mathrm{n}^{\mathrm{c}}$ & $3.6 \pm 0.9$ & $(0.4)$ & $1435^{f}$ & $-51^{i}$ \\
\hline HDE $237019 \ldots \ldots$ & $137.42,-1.07$ & $\mathrm{O} 8 \mathrm{~V}^{\mathrm{a}}$ & $2.4 \pm 0.4$ & $(0.3)$ & $(1800)$ & $-25^{\mathrm{b}}$ \\
\hline HD $16691 \ldots \ldots \ldots$ & $137.72,-2.73$ & O 4 If $+^{c}$ & $3.4 \pm 0.9$ & $\begin{array}{l}7^{\mathrm{h}} \\
18\end{array}$ & $(2300)$ & $-114^{\mathrm{g}},-46^{\mathrm{d}}$ \\
\hline HD $16832 \ldots \ldots \ldots$ & $138.00,-2.88$ & $\mathrm{O} 9.5 \mathrm{II}^{\mathrm{c}}$ & $2.6 \pm 0.7$ & $\begin{array}{l}10 \\
(0.4)\end{array}$ & (1500) & \\
\hline
\end{tabular}

ReFERENCES.- (a) Mathys 1989; (b) Kendall et al. 1995; (c) Walborn 1973; (d) Cruz-Gonzalez et al. 1974; (e) Chlebowsky \& Garmany 1991; (f) Howarth et al. 1997; (g) Conti et al. 1977; (h) Leitherer 1988; (i) McLachlan \& Nandy 1985; (j) Underhill \& Gilroy 1990; (k) Scuderi et al. 1998; (1) Lamers \& Leitherer 1993; (m) Groenewegen \& Lamers 1989; (n) Puls et al. 1996; (o) Walborn 1972. 
TABLE 2

Parameters of the 21 cm Line Observations

\begin{tabular}{lc}
\hline \hline Parameter & Value \\
\hline Main beam efficiency ............. & 0.72 \\
Beam size FWHM (arcmin) $\ldots \ldots \ldots$ & 9 \\
Surveyed area .................. & $132.5 \leq l \leq 139.0$ \\
& $-4.0 \leq b \leq+0.2$ \\
Total bandwidth $(\mathrm{MHz}) \ldots \ldots \ldots \ldots$ & 3.125 \\
Velocity range $\left(\mathrm{km} \mathrm{s}^{-1}\right) \ldots \ldots \ldots \ldots$ & $-430,+230$ \\
Velocity resolution $\left(\mathrm{km} \mathrm{s}^{-1}\right) \ldots \ldots$ & 1.3 \\
Integration time per point $(\mathrm{s}) \ldots \ldots$ & 31 \\
Effective integration time $(\mathrm{s}) \ldots \ldots \ldots$ & 63 \\
\hline
\end{tabular}

Kalberla, Mebold, \& Reif 1982). All profiles were corrected for stray radiation using the procedure developed by Kalberla, Mebold, \& Reich (1980). To increase the signal-tonoise ratio we have averaged both polarizations. The final rms-noise temperature is about $0.14 \mathrm{~K}$.

In order to investigate the large scale $\mathrm{H}$ I gas distribution in the environs of the Per OB1 association, complementary $\mathrm{H}$ I data were taken from the Leiden Dwingeloo H I Survey by Hartman \& Burton (1997). This survey, obtained with the Dwingeloo radio telescope, covers a regular grid of 0.5 both in $l$ and $b$, with a HPBW of 0.6 , sampled at $1.03 \mathrm{~km}$ $\mathrm{s}^{-1}$. The rms noise is about $0.07 \mathrm{~K}$ in brightness temperature.

Additional data sets were collected from other sources. To analyze the distribution of the ionized gas in the region we used the radio continuum survey at $2.7 \mathrm{GHz}$ obtained with an angular resolution of 4:3 (Fürst et al. 1990). ${ }^{2}$ The distribution of the molecular material was investigated using the FCRAO CO Survey of the Outer Galaxy (Heyer et al. 1998) available at the NASA ADC. The HPBW of this survey is $45^{\prime \prime}$ sampled every $22^{\prime \prime}$. The velocity ranges from -153 to $+40 \mathrm{~km} \mathrm{~s}^{-1}$ sampled every $0.81 \mathrm{~km} \mathrm{~s}^{-1}$ with a velocity resolution of $0.98 \mathrm{~km} \mathrm{~s}^{-1}$. The dust emission distribution was analyzed through IRAS images (angular resolution $\approx 6^{\prime}$ ) available at the NASA ADC.

\section{THE LARGE SCALE H I DISTRIBUTION IN THE REGION OF PER OB1}

To facilitate the analysis of the $\mathrm{H} \mathrm{I}$ emission distribution, in Figure 1 we show the average $\mathrm{H} \mathrm{I} 21 \mathrm{~cm}$ line spectrum for the region $133.0 \leq l \leq 138.0,-3.5 \leq b \leq 0.0$ obtained from the Effelsberg observations. The profile displays the velocity interval -110 to $+20 \mathrm{~km} \mathrm{~s}^{-1}$ where significant emission is detected.

The $\mathrm{H}$ I spectrum shows three main components. H I emission that peaks near $v \simeq-4 \mathrm{~km} \mathrm{~s}^{-1}$ is associated with the local spiral arm at distances $d_{k} \leq 1.5-2 \mathrm{kpc}$. The component that peaks at $v \simeq-45 \mathrm{~km} \mathrm{~s}^{-1}$ appears related to the Perseus spiral arm at $d_{k} \simeq 2.0-7 \mathrm{kpc}$ (e.g., Caswell \& Haynes 1987); while the features with velocities $v \leq-75$ $\mathrm{km} \mathrm{s}^{-1}$ would correspond to the Cygnus spiral arm (Kimeswenger \& Weinberger 1989) at $d_{k} \geq 10 \mathrm{kpc}$. The kinematical distances were estimated from the analytical fit to the rotation curve obtained by Brand \& Blitz (1993).

To investigate the neutral gas distribution around $\mathrm{Per}$ OB1, we examined the $\mathrm{H}$ I emission in the region $128.0 \leq$

\footnotetext{
${ }^{2}$ Available at: http://www.mpifr-bonn.mpg.de/survey.html.
}

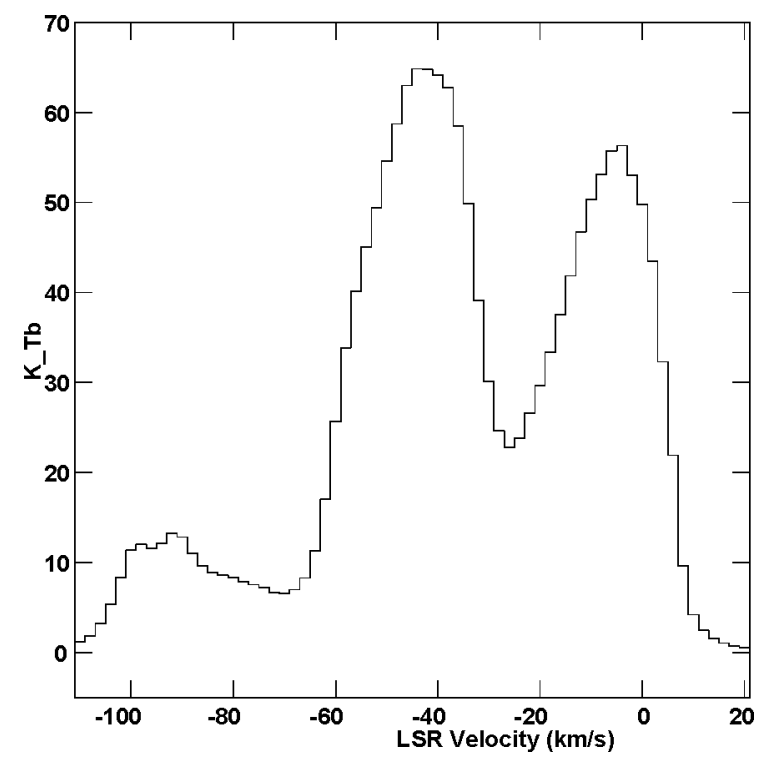

FIG. 1.-Average $\mathrm{H}$ I profile obtained from the Effelsberg observations through the region $133^{\circ} 0 \leq l \leq 138^{\circ} 0,-3.5 \leq b \leq 0.0$.

$l \leq 142.0,-17.0 \leq b \leq+1.0$. The left panel of Figure 2 illustrates the neutral hydrogen column density distribution $N_{\mathrm{H} \text { I }}$ within the velocity range -32 to $-20 \mathrm{~km} \mathrm{~s}^{-1}$, under the assumption of optically thin emission, obtained from the low angular resolution $\mathrm{H}$ I survey. The $\mathrm{O}$ and $\mathrm{B}$ stars belonging to Per OB1 are indicated as asterisks and plus signs, respectively. The circles mark the position of the open clusters $h$ and $\chi$ Persei. For the sake of clarity, only the position of the O-type star HD 14434 is shown in this region.

The map shows a region of low emission centered at $(l, b)=(134.5,-2.5)$, of about $6^{\circ}$ in size. Since it is detected within the velocity range -32 to $-20 \mathrm{~km} \mathrm{~s}^{-1}$, we adopt the mean velocity in the interval $\left(-26 \mathrm{~km} \mathrm{~s}^{-1}\right)$ as the systemic velocity $v_{\text {sys }}$. The application of a flat galactic rotation curve (Brand \& Blitz 1993) predicts that gas with $v_{\text {sys }}$ should be located at a kinematic distance $d_{k} \simeq 2.5 \mathrm{kpc}$. Their Figures $2 a$ and $2 b$, which include noncircular motions, indicate $d_{k} \simeq 1.8 \mathrm{kpc}$ for the same velocity. These values are in agreement with the optical distance to Per OB1 $(2.3 \mathrm{kpc}$; see $\S 1$ ), suggesting that the $\mathrm{H}$ I feature may be related to Per OB1.

The $\mathrm{H}$ I low-emission region appears to be excavated in the gas layer. It is partially encircled by enhanced emission regions seen at $l \approx 132.5$, from $b \approx-4.0$ to $0^{\circ} ; b=0^{\circ}$ from $l \approx 133.0$ to 136.0 and from $(l, b)=\left(136.0,0^{\circ}\right)$ to $(l, b)=$ $(139.0,-4.0)$. The contour lines seem to be piled up mainly at $b=0^{\circ}$ from $l=133.0$ to $135^{\circ} 0$, and $l=132.5$ from $b=$ -3.0 to -1.0 , suggesting that the neutral gas is compressed. Contrariwise, a smooth gradient is present at $l=136^{\circ}$. This structure can be identified with the H I shell candidate described by Heiles (1979) and related to Per OB1. Note that most of the early-type stars in the association appear projected onto the low-emission region or onto their borders. Thus, the spatial coincidence between the $\mathrm{H}$ i low-emission region and Per OB1, suggests that the $\mathrm{OB}$ association is interacting with its environs and that the low-emission region and the surrounding envelope may be the result of such interaction. We can derive an upper limit of $10 \mathrm{~km} \mathrm{~s}^{-1}$ for the expansion velocity of this structure. 

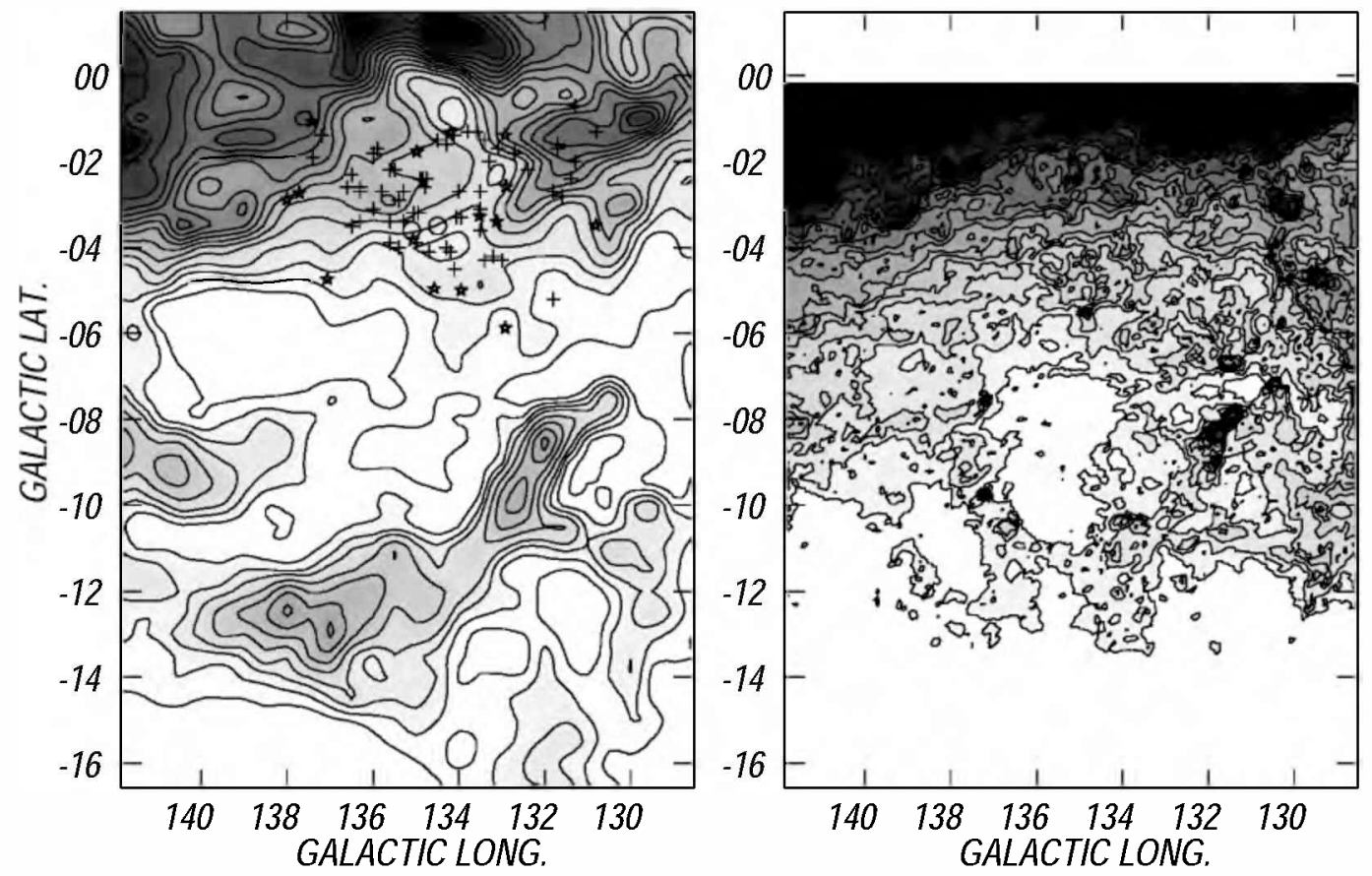

FIG. 2. - Left panel: Latitude-longitude $\mathrm{H}$ I column density image through the velocity range -32 to $-20 \mathrm{~km} \mathrm{~s}^{-1}$, obtained from the Hartman \& Burton survey, showing the $\mathrm{H}$ I gas distribution in the vicinity of Per OB1. The gray scale corresponds to $(1.7-12.5) \times 10^{20} \mathrm{~cm}^{-2}$. The contour levels are $1.5,2.0,2.5$, $3.0,3.8,4.6,5.4,6.2,7.0,7.8,8.6,9.4,10.2,11.0$, and $11.8 \times 10^{20} \mathrm{~cm}^{-2}$. The position of the O-type stars belonging to Per OB1 is indicated with stars, while the B0-B5 type stars are shown as plus signs. The small circles near $b=-4^{\circ}$ mark the position of the open clusters. Right panel: IRAS image at $100 \mu \mathrm{m}$. The gray scale corresponds to $9-45 \mathrm{MJy} \mathrm{sr}^{-1}$. The contour levels are $10,12,14,16,18,20,22,25,28,31,34,37$, and $40 \mathrm{MJy} \mathrm{sr}^{-1}$.

The figure also illustrates the presence of strong $\mathrm{H}$ i emission between $-14 \leq b \leq-8^{\circ}$, where the brighter $\mathrm{H}$ I clouds define an arclike feature. An inspection of the $\mathrm{H} \mathrm{I}$ maps at different velocities shows the presence of strong $\mathrm{H} \mathrm{I}$ emission at $-18^{\circ} \leq b \leq-8^{\circ}$, within the velocity interval -55 to $-18 \mathrm{~km} \mathrm{~s}^{-1}$, which would be related to the Perseus spiral arm. The large arclike feature at $(l, b)=(134.0$, $-12.0)$ is clearly detected within the velocity interval -33 to $-20 \mathrm{~km} \mathrm{~s}^{-1}$. Part of this feature can be identified with the worm candidate GS132.6-9. The brightness of the arclike feature vanishes toward higher negative velocities until it disappears at $\simeq-39 \mathrm{~km} \mathrm{~s}^{-1}$. We believe that the arclike structure also conforms the $\mathrm{H}$ i shell related to Per OB1.

The IRAS image at $100 \mu \mathrm{m}$ (Fig. 2, right panel) reveals infrared emission following the inner border of the large $\mathrm{H} \mathrm{I}$ arclike feature. Slightly enhanced IR emission also delineates the section of the $\mathrm{H}$ I shell seen at $l \geq 137.0$, from $b=-9.5$ to -5.0 . Enhanced IR emission is also present at $(l, b)=\left(137^{\circ} .0,-10.0\right)$, where little $\mathrm{H} \mathrm{I}$ is detected. Thus, an IR counterpart can be identified in the region of the shell toward higher negative Galactic latitudes. The IR counterpart of the region of the $\mathrm{H}$ i shell closer to the galactic plane is more difficult to distinguish. It may be masked by the bulk of the galactic contribution.

The radio continuum emission distribution at $1408 \mathrm{MHz}$ (Reich et al. 1990) displays a region of low emission coincident with the large cavity seen in the IRAS and $\mathrm{H}$ I images near $(l, b)=\left(135^{\circ} 0,-8.5\right)$. A relatively small emission region at $(l, b)=(131.5,-8.3)$, which correlates with the brightest region of the $\mathrm{H} \mathrm{I}$ arclike feature, is also observed.

Adopting for the $\mathrm{H}_{\mathrm{I}}$ shell the optical distance to Per OB1, the angular and linear radius of the upper part of the shell (i.e., the region closer to the galactic plane) is $\simeq 3.0$ or
$110 \mathrm{pc}$. By including the arclike feature and the emission seen near $(l, b)=\left(141^{\circ} 0,-9^{\circ}\right)$, the shell related to Per OB1 is $9^{\circ} \times 14^{\circ}$ or $350 \times 550 \mathrm{pc}$ in size (which corresponds to a mean radius of $220 \mathrm{pc}$ ). The uncertainty in size is $20 \%$, assuming an error of $20 \%$ in the distance to Per OB1.

The neutral masses in both regions of the shell were evaluated separately. We estimated the swept-up mass in the upper part of the shell $M_{\mathrm{s}}$ from mean $N_{\mathrm{II}}$-values in the low-emission region and in their surroundings and adopted the swept-up mass to be a mean value between them. We found $M_{\mathrm{s}} \simeq 3.7 \times 10^{4} M_{\odot}$. A helium abundance of $10 \%$ was assumed. The mass content in the lower part of the shell is about $5.1 \times 10^{4} M_{\odot}$. These masses are lower limits since we have taken into account the neutral mass only. By distributing $8.8 \times 10^{4} M_{\odot}$ uniformly within a sphere of $220 \mathrm{pc}$ in radius, the ambient gas density results $\sim 0.1 \mathrm{~cm}^{-3}$. The error in the derived masses is about $40 \%$.

No $\mathrm{H}$ II region was cataloged within the area of Per OB1. A radio recombination line was detected at $l=132.16, b=$ -0.73 , at $v_{\mathrm{LSR}}=-56.6 \mathrm{~km} \mathrm{~s}^{-1}$ (Lockman 1989). This region is projected onto the border of the $\mathrm{H}$ I shell and is located at a kinematic distance of 5-6 kpc. Because of its position and distance, we assume it is unrelated to Per OB1.

\section{H I BUBBLES SURROUNDING MASSIVE STARS}

To investigate the $\mathrm{H}$ I gas distribution in the environs of the $\mathrm{O}$ and $\mathrm{O}$ stars in the association we used the $\mathrm{H}$ I data obtained at Effelsberg. $\mathrm{H}$ I cavities and shells candidates to be related to the individual stars in the association were carefully searched for in a series of $(l, b)$ maps constructed in steps of $2 \mathrm{~km} \mathrm{~s}^{-1}$, showing brightness temperature at constant velocity. We have discovered $\mathrm{H}$ I structures probably associated with the Of stars HD 14947, 14442, and 16691, with the O stars HD 13022 and HD 13338, and with the B 
stars HD 15233 and Hilt 311. In all cases, the location of the stars within the $\mathrm{H}$ I cavities or on their borders and the agreement between the kinematic distance to the features and the optical distance derived for Per OB1 indicate an association between the $\mathrm{H}$ I structure and the star.

The structures are partially seen in Figure 3, which displays the $\mathrm{H}$ I gas distribution within the same velocity interval as Figure 2, as obtained using the Effelsberg $\mathrm{H}$ i data. The image shows the same large-scale feature as Figure 2 (left panel) and a large number of small-scale $\mathrm{H}$ i structures with some of the massive stars in the association projected onto small $\mathrm{H}$ I voids.

The main physical parameters of the $\mathrm{H}$ I structures are listed in Table 3 . The velocity interval $v_{1}, v_{2}$ corresponds to the velocity range in which each $\mathrm{H}$ I feature is clearly identified and is usually larger than the velocity range where the column density image was obtained. The systemic velocity $v_{\text {sys }}$ of each shell was used to derive its kinematic distance $d_{k}$. Since, within errors, $d_{k}$ agrees in all cases with the distance to Per OB1, we adopted the optical distance to the association $(2.3 \mathrm{kpc})$ in our calculations, instead of the kinematic distances to the $\mathrm{H}$ I structures or the stellar distances. The radius $R_{s}$ of each structure was obtained as a mean value between the radii of the void $\left(R_{v}\right)$ and the envelope $\left(R_{\mathrm{env}}\right)$,

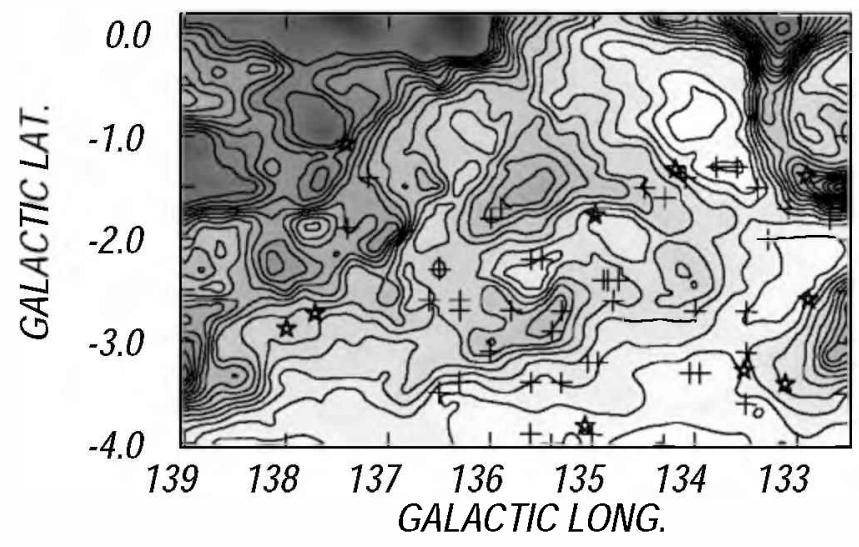

FIG. 3. - Same as Fig. 2, as obtained from the Effelsberg data. The gray scale corresponds to $(2.4-13.6) \times 10^{20} \mathrm{~cm}^{-2}$. The contour levels are 2.0 , $2.5,3.0,3.5,4.0,4.5,5.0,5.5,6.0,6.5,7.0,7.5,8.0,8.5,9.0,9.5$, and $10.0 \times 10^{20} \mathrm{~cm}^{-2}$. The star and plus signs have the same meaning as in Fig. 2 . and corresponds approximately to the radius defined by the position of the maxima in the shell. The major and minor semiaxis $\left(r_{M} \times r_{m}\right)$ are given for cases of noncircular cavities and shells. The neutral masses were evaluated assuming the optically thin case and a typical interstellar helium abundance of $10 \%$. The swept-up neutral masses $M_{s}$ were obtained as mean values between the neutral mass deficiency in the voids $M_{\text {def }}$ and the mass in the envelopes $M_{\text {env }}$. As in previous papers, ambient densities were evaluated by distributing $M_{s}$ over the volume of the bubbles. The expansion velocities were derived as $\left(v_{1}-v_{2}\right) / 2+2 \mathrm{~km} \mathrm{~s}^{-1}$. Swept-up masses and expansion velocities were used to estimate the kinetic energies of the neutral shells.

The uncertainty in $v_{\mathrm{sys}}$, which is $2 \mathrm{~km} \mathrm{~s}^{-1}$, arises in the velocity interval between the individual maps in the series; while the uncertainty in $d_{k}$ originates in the presence of noncircular motions of about $\pm 7 \mathrm{~km} \mathrm{~s}^{-1}$ (Burton 1988). The major source of error in radii, masses and kinetic energies originates in the distance uncertainty. An error of $20 \%$ in the distance to Per OB1 (see $\S 1$ ) implies $20 \%$ error in radius and $40 \%$ error in the derived masses. The uncertainty in the kinetic energy is at least $45 \%$.

In the following sections we briefly describe the discovered $\mathrm{H}$ I bubbles.

\subsection{The ISM in the Vicinity of HD $14442[O 5 n(f) p]$}

The top panel of Figure 4 displays the $\mathrm{H}$ I column density distribution within the velocity interval -28 to $-18 \mathrm{~km}$ $\mathrm{s}^{-1}$ in the neighborhood of HD 14442. The O5n(f) star, indicated by a star, appears projected close to the borders of a large and complex $H$ I cavity centered at $(l, b)=(134.1$, $-0.9)$. The cavity contains two minima, centered at $(l, b)=$ $(134.0,-0.9)$ and $(l, b)=(134.4,-0.5)$. The surrounding envelope is clearly detected toward the southern border of the cavity $(b=-1.7)$. Toward $l \approx 133.25$, the $\mathrm{H}$ I envelope may be masked by the presence of a $\mathrm{H}$ I filament probably unrelated to the O-type star. The existence of a $\mathbf{H}$ I shell bordering the cavity near $l \approx 135.0$ cannot be confirmed from the present data. The portion of the $\mathrm{H}$ I shell toward $b=-0.25$, which is barely visible, separates the doubleminima structure from a smaller cavity detected at $(l, b)=$ $\left(135^{\circ} .2,-0.0\right)$.

Adopting the mean value of the velocity interval in which the $\mathrm{H} \mathrm{I}$ feature is seen as the systemic velocity $v_{\text {sys }}=-23$ $\mathrm{km} \mathrm{s}^{-1}$, we derived a kinematic distance of $\simeq 2.2 \mathrm{kpc}$, in

TABLE 3

Parameters of the H i Structures Surrounding Massive Stars

\begin{tabular}{|c|c|c|c|c|c|c|}
\hline Star & HD 14442 & HD 14947 & HD 16691 & HD13022 & HD13338 & $\begin{array}{c}\text { HD15233 } \\
\text { Hilt } 311\end{array}$ \\
\hline$(l, b)$ center $\ldots \ldots \ldots \ldots \ldots \ldots \ldots . . . . . . . .$. & $134.1,-0.9$ & $134.7,-1.8$ & $137.65,-3.15$ & $133.15,-2.25$ & $133.75,-3.3$ & $135.6,-2.3$ \\
\hline Vel. interval $v_{1}, v_{2}\left(\mathrm{~km} \mathrm{~s}^{-1}\right) \ldots \ldots$ & $-32,-18$ & $-30,-14$ & $-32,-20$ & $-38,-14$ & $-26,-16$ & $-32,-16$ \\
\hline Systemic vel. $v_{\text {sys }}\left(\mathrm{km} \mathrm{s}^{-1}\right) \ldots \ldots$ & -23 & -22 & -25 & -22 & -21 & -22 \\
\hline Kinematic distance $d_{k}(\mathrm{kpc}) \ldots \ldots$ & 2.2 & 2.1 & 2.4 & 2.1 & 2.0 & 2.1 \\
\hline Radius of the cavity (pc) .......... & $30 \times 18$ & $16 \times 8$ & $26 \times 15$ & $22 \times 15$ & 15 & 12 \\
\hline Radius of the envelope (pc) ...... & 40 & $30 \times 20$ & $37 \times 25$ & $\ldots$ & 22 & 24 \\
\hline Mean radius $R_{\mathrm{s}}(\mathrm{pc}) \ldots \ldots \ldots \ldots$ & $35 \times 30$ & $24 \times 14$ & $31 \times 20$ & $22 \times 29$ & 18 & 18 \\
\hline$M_{\text {der }}\left(M_{\odot}\right) \ldots \ldots \ldots \ldots \ldots \ldots \ldots \ldots \ldots$ & 920 & 180 & 620 & 1100 & 240 & 220 \\
\hline$M_{\text {env }}\left(M_{\odot}\right) \ldots \ldots \ldots \ldots \ldots \ldots \ldots$ & 4060 & 1900 & 1660 & $\ldots$ & 700 & 1300 \\
\hline$M_{s}\left(M_{\odot}\right) \ldots \ldots \ldots \ldots \ldots \ldots \ldots \ldots \ldots$ & 2500 & 1040 & 1140 & 1100 & 470 & 750 \\
\hline$n\left(\mathrm{~cm}^{-3}\right) \ldots \ldots \ldots \ldots \ldots \ldots \ldots \ldots \ldots \ldots$ & 0.8 & 1.8 & 1.0 & 1.4 & 0.8 & 1.3 \\
\hline$V_{\text {exp }}\left(\mathrm{km} \mathrm{s}^{-1}\right)$ & 9 & 10 & 8 & 14 & 7 & 10 \\
\hline$E_{k}\left(10^{48} \mathrm{ergs}\right) \ldots$ & 2.0 & 1.0 & 0.7 & $\geq 1.6$ & 0.3 & 0.8 \\
\hline
\end{tabular}



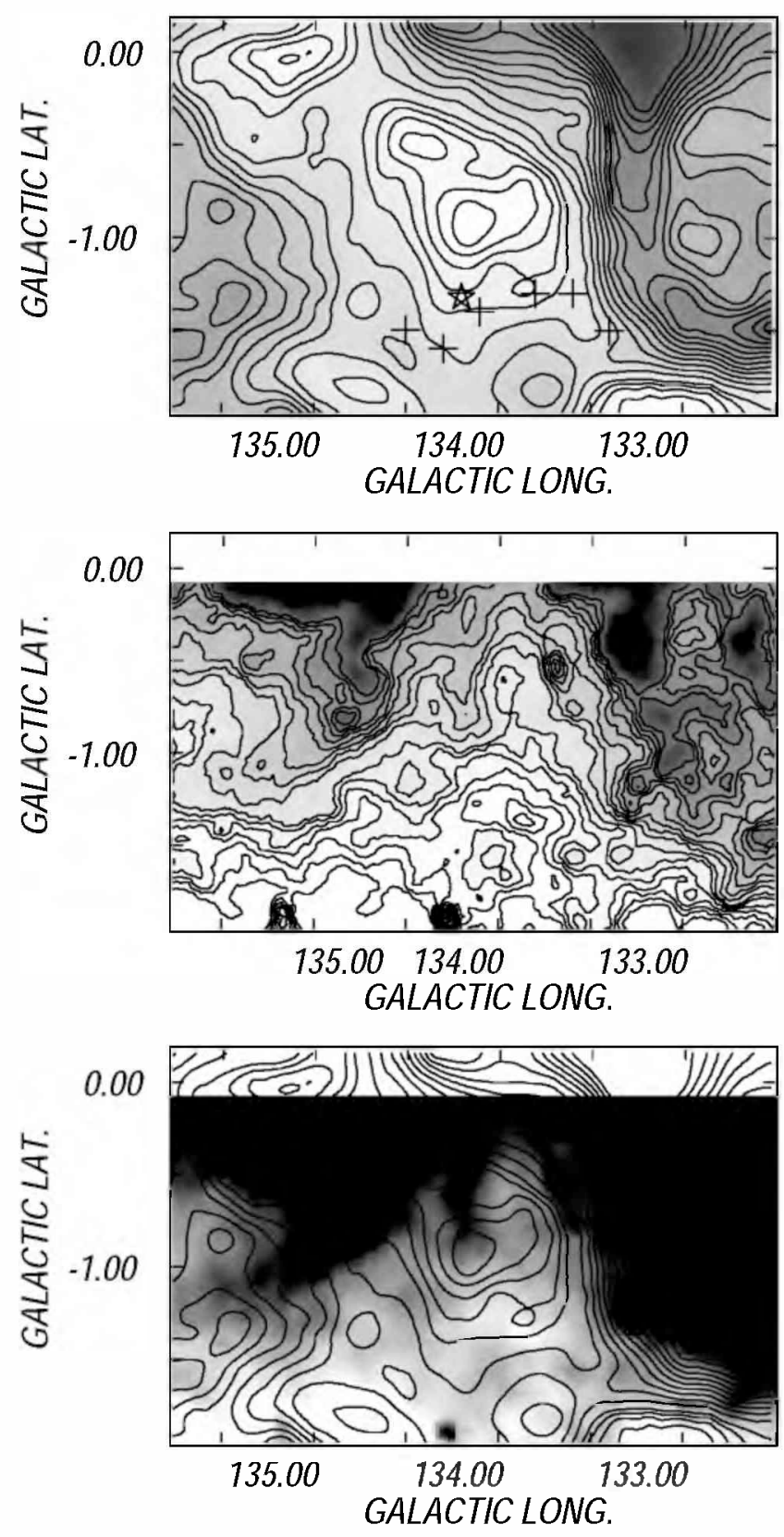

FIG. 4.-Top panel: Latitude-longitude $\mathrm{H}$ I column density image through the velocity range -28 to $-18 \mathrm{~km} \mathrm{~s}^{-1}$, showing the $\mathrm{H}$ I gas distribution in the vicinity of HD 14442. The gray scale corresponds to $(2.2-9.5) \times 10^{20} \mathrm{~cm}^{-2}$. The contour levels are $1.8,2.02 .2,2.4,2.6,2.8,3.0$, $3.4,3.8,4.2,4.6,5.0,5.4,5.8,6.2,6.6,7.0,7.4,7.8$, and $8.2 \times 10^{20} \mathrm{~cm}^{-2}$. The star indicates the position of HD 14442, while the plus signs mark the position of other early-type stars (see $\$ 5$ ). Middle panel: IR image at 100 $\mu \mathrm{m}$. The gray scale corresponds to 40 to $120 \mathrm{MJy} \mathrm{sr}^{-1}$ and the contour lines are $36,38,40,42,44,46,48,52,56,60,66,72,78,84$, and $90 \mathrm{MJy} / \mathrm{sr}$. Bottom panel: Overlay of the same $\mathrm{H}$ I contour lines and a gray-scale IR $A S$ image. The gray scale corresponds to 36 to $60 \mathrm{MJy} \mathrm{sr}^{-1}$.

agreement with the optical distance to Per OB1 and with the stellar distance.

The middle panel of Figure 4 presents the IRAS infrared emission at $100 \mu \mathrm{m}$, while the bottom panel displays an overlay of the IR (gray scale) and H I (contour lines) images. The $\mathrm{H}$ I minimum at $(l, b)=(134.4,-0.5)$ appears projected onto a region of relatively low IR emission delineated by enhanced IR emission toward $b=-0.5$ and $l=134.5$. The strong IR emission detected toward $l=133.0$ is discon- nected to the cavity. The lowest IR emission region is detected near $(l, b)=(133.8,-1.35)$, projected onto a small region of the $\mathrm{H}$ I minimum at $b=-0.9$. The lack of a clear IR shell might be due to the presence of a galactic IR gradient and confusion effects originated in the fact that IR emission prevents discrimination of material at different velocities.

Very faint $\mathrm{H} \alpha+[\mathrm{N}$ II $]$ emission (Parker, Gull, \& Kirschner 1979) is seen projected onto part of the H I cavity, suggesting the presence of low density ionized gas. The radio continuum emission at $2.7 \mathrm{GHz}$ (Fürst et al. 1990) is quite complex in this region. An area of low emission centered at the position of the lowest IR emission region described before is clearly visible. No $\mathrm{CO}$ emission was detected within the velocity range where the $\mathrm{H}$ I structure is present.

The distribution of material in the surroundings of the Of star supports the idea that this material could have been swept-up by the action of the strong stellar winds of the star. Both radio continuum and IR observations suggest that only the $\mathrm{H}$ I cavity with the highest negative galactic latitude could have been blown by the Of star.

The plus signs in Figure 4 mark the position of the earlytype stars belonging to Per OB1 which are projected onto the borders of the $\mathrm{H}$ I cavity, namely $\mathrm{BD}+59^{\circ} 451$ (B1II), $\mathrm{BD}+59^{\circ} 456(\mathrm{~B} 0.5 \mathrm{~V}), \mathrm{BD}+59^{\circ} 461(\mathrm{~B} 1 \mathrm{II}), \mathrm{BD}+58^{\circ} 451$ (B1III), BD $+58^{\circ} 453$ (B1V), and HDE236960 (B0.5III). Their contribution in shaping the cavity will be analyzed in $\S 5$.

\subsection{The ISM surrounding HD 14947 [O5If +$]$}

The $\mathrm{H}$ I column density distribution within the velocity interval -28 to $-14 \mathrm{~km} \mathrm{~s}^{-1}$ in the vicinity of the O5f + star is illustrated in the top panel of Figure 5. An elongated cavity, centered at $(l, b)=(134.7,-1.8)$ is clearly seen in the image. HD 14947, indicated by a star symbol, appears projected within the cavity, close to one of its higher density borders. The void is encircled by an almost complete and thick shell. An inspection of the $\mathrm{H} \mathrm{I}$ images at full velocity resolution shows that as the velocity changes, the position of the center of the $\mathrm{H}$ I structure varies, indicating a distorted feature. Within the velocity interval -30 to $-22 \mathrm{~km}$ $\mathrm{s}^{-1}$ the minimum is centered at $(l, b)=(134.7,-2.0)$, while within the velocity interval -22 to $-14 \mathrm{~km} \mathrm{~s}^{-1}$, it is centered at $(l, b)=(134.7,-1.7)$. Adopting a systemic velocity $v_{\text {sys }}=-22 \mathrm{~km} \mathrm{~s}^{-1}$, the kinematic distance turns out to be $2.1 \mathrm{kpc}$, in very good agreement with the distance to Per $\mathrm{OB} 1$ as well as with the stellar distance.

The location of HD 14947 close to one of the higher density borders of the cavity together with the agreement between the kinematic distance of the structure and the stellar distance suggest a physical association between the $\mathrm{H}$ I structure and the Of star. No other massive star belonging to the association appears within the void or onto its borders.

The bottom panel of Figure 5 displays the same H I contours superposed to a gray-scale representation of the IRAS image at $100 \mu \mathrm{m}$. The image reveals a low IR emission region, which coincides with the $\mathrm{H}$ I cavity and is surrounded by an open ringlike feature opposite the galactic plane.

An inspection of the radio continuum image at $2.7 \mathrm{GHz}$ reveals the presence of weak diffuse emission within the region of the cavity. However, it is not clear if it is related to 

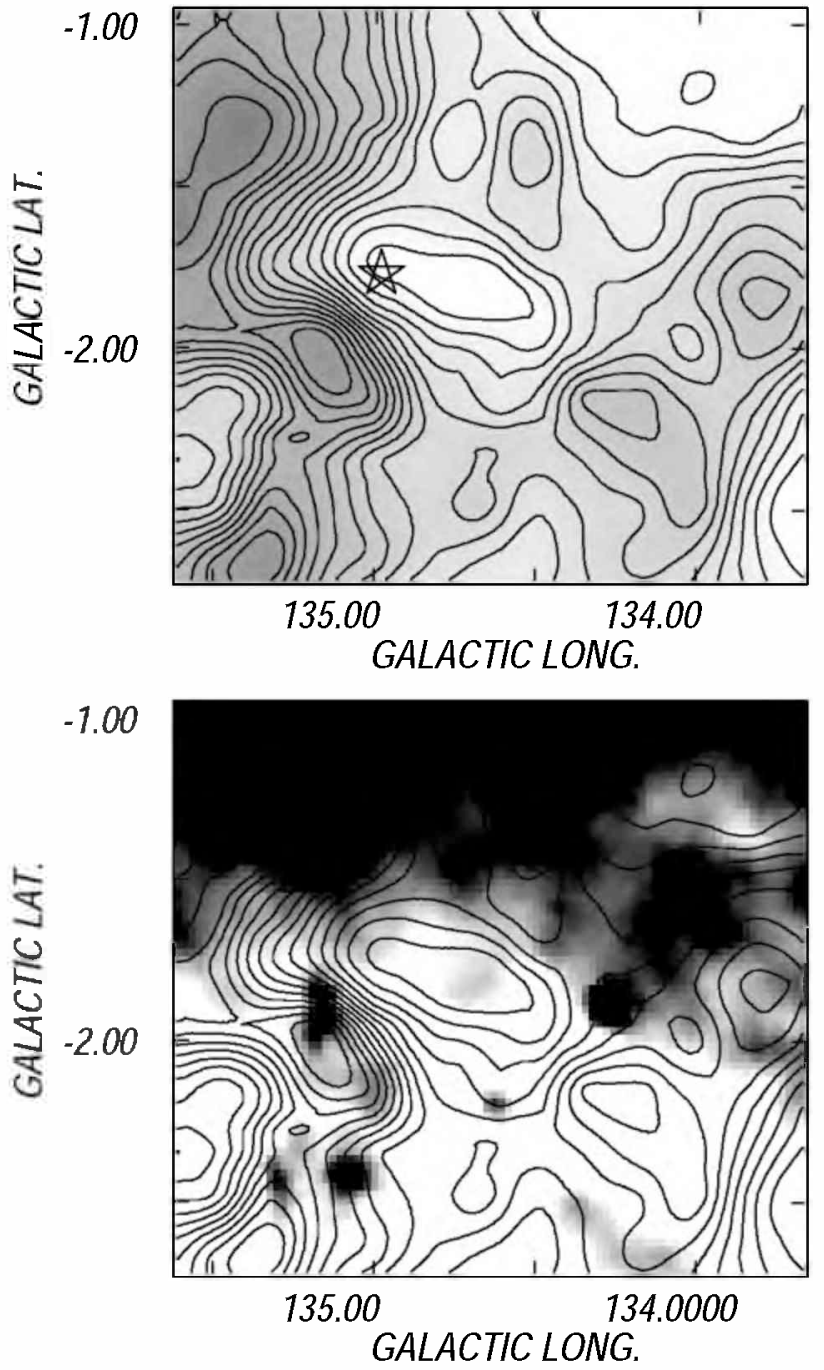

FIG. 5.-Top panel: Latitude-longitude $\mathrm{H}_{\mathrm{I}}$ column density image through the velocity range -28 to $-14 \mathrm{~km} \mathrm{~s}^{-1}$, showing the $\mathrm{H}$ I gas distribution in the vicinity of HD 14947. The gray scale corresponds to $(3.3-6.5) \times 10^{20} \mathrm{~cm}^{-2}$. The contour levels are $3.4,3.6,3.8,4.0,4.2,4.4,4.6$, $4.8,5.0,5.2,5.4,5.6,5.8,6.0,6.2,6.6$, and $7.0 \times 10^{20} \mathrm{~cm}^{-2}$. The star indicates the position of HD 14947. Bottom panel: Overlay of the same H I contour lines and a gray-scale IRAS image at $100 \mu \mathrm{m}$. The gray scale corresponds to $35-42 \mathrm{MJy} \mathrm{sr}^{-1}$.

the $\mathrm{H}$ I structure. The analysis of the molecular data in the region does not show molecular material associated with the detected $\mathrm{H}$ i shell.

As for the case of HD 14442, these results support the idea of a wind-blown bubble origin for the $\mathrm{H}$ I structure linked to HD 14947.

\subsection{The ISM in the Vicinity of HD 16691 [O4If +$]$}

The top panel of Figure 6 displays the H I column density distribution around HD 16691, within the velocity interval -28 to $-22 \mathrm{~km} \mathrm{~s}^{-1}$. The image shows a low-emission region centered at $(l, b)=(137.65,-3.15)$. It appears open opposite to the galactic plane, probably showing the effects of the galactic density gradient. The O4If + star, indicated as a star symbol, is located close to one of the high density borders of the feature. The brightest portions of the shell are detected toward the galactic plane and toward the higher galactic longitude border. Assuming $v_{\mathrm{sys}}=-25 \mathrm{~km} \mathrm{~s}^{-1}$, a
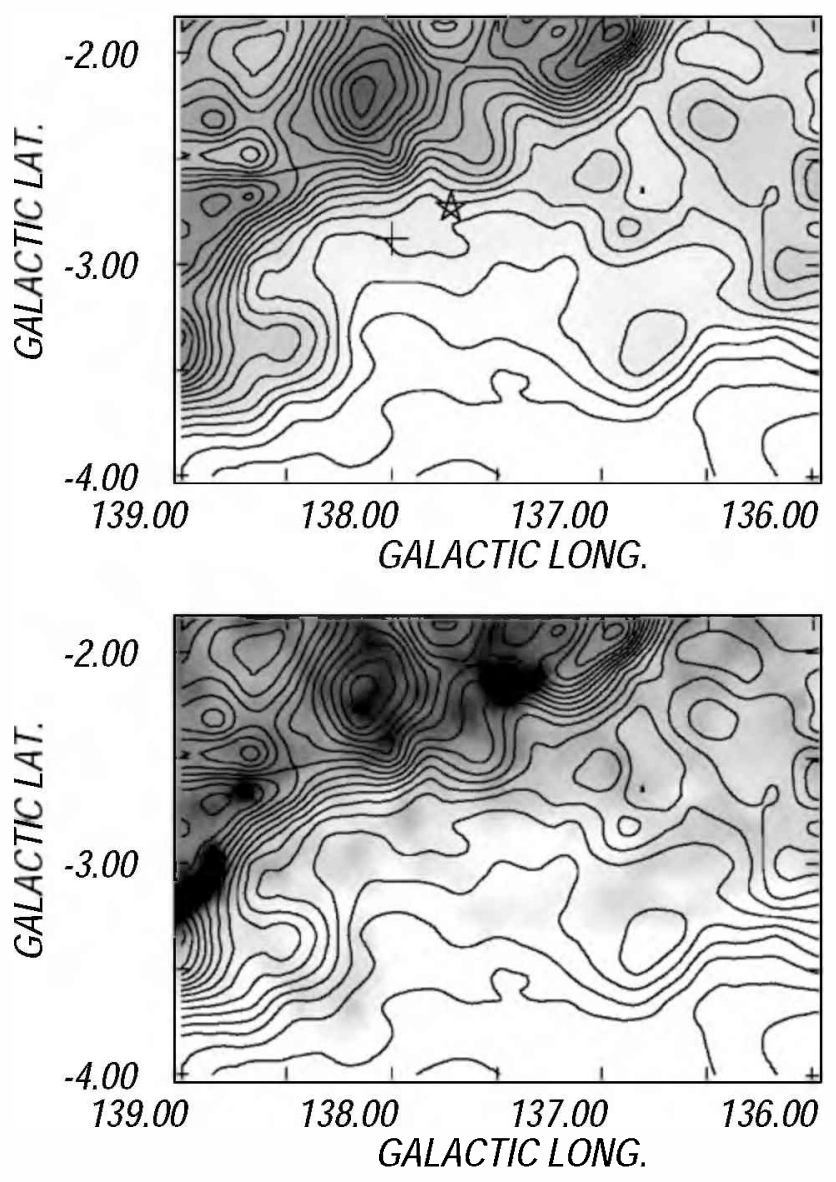

FIG. 6.-Top panel: Latitude-longitude $\mathrm{H}$ I column density image through the velocity range -28 to $-22 \mathrm{~km} \mathrm{~s}^{-1}$, showing the $\mathrm{H}$ I gas distribution in the vicinity of HD 16691 . The gray scale corresponds to $(1.8-4.8) \times 10^{20} \mathrm{~cm}^{-2}$. The contour levels are from 1.1 to $4.7 \times 10^{20} \mathrm{~cm}^{-2}$ in steps of $0.2 \times 10^{20} \mathrm{~cm}^{-2}$. The star indicates the position of HD 16691, while the plus sign marks the position of HD 16832 (see $\S 5$ ). Bottom panel: Overlay of the same $\mathrm{H}$ I contour lines and a gray scale IRAS image at 100 $\mu \mathrm{m}$. The gray scale corresponds to $28-50 \mathrm{MJy} \mathrm{sr}^{-1}$.

kinematic distance of about $2.4 \mathrm{kpc}$ is derived, in agreement with the distance to Per OB1. The cross marks the position of the O9.5II star HD 16832 (see Table 1). According to its location, it might also contribute to the formation of the cavity.

The bottom panel of Figure 6 shows the superposition of the $100 \mu \mathrm{m}$ IRAS image (gray scale) and the $\mathrm{H}$ I emission (contour lines). A clear IR counterpart is detected. The radio continuum emission at $2.7 \mathrm{GHz}$, which is very faint in this area, displays a low-emission region which partially correlates with the $\mathrm{H}$ I cavity. No CO emission was found to be associated with this structure.

In this case, the evidence of the presence of an $\mathrm{H}$ I structure related to the star is less conclusive than in the other cases. However, we note that similar $\mathrm{H}$ I distributions have been found for some $\mathrm{H}$ I bubbles associated with WR stars (see Arnal et al. 1999).

\subsection{The ISM in the Vicinity of HD $13022[09.5 I I-I I I((n))]$ and $H D 13338$ [O9.5V]}

The neutral gas distribution in the vicinity of these O-type stars is shown in Figure 7, where we display the $N_{\mathrm{H}}$ within the velocity interval -24 to $-16 \mathrm{~km} \mathrm{~s}^{-1}$. HD 13022 

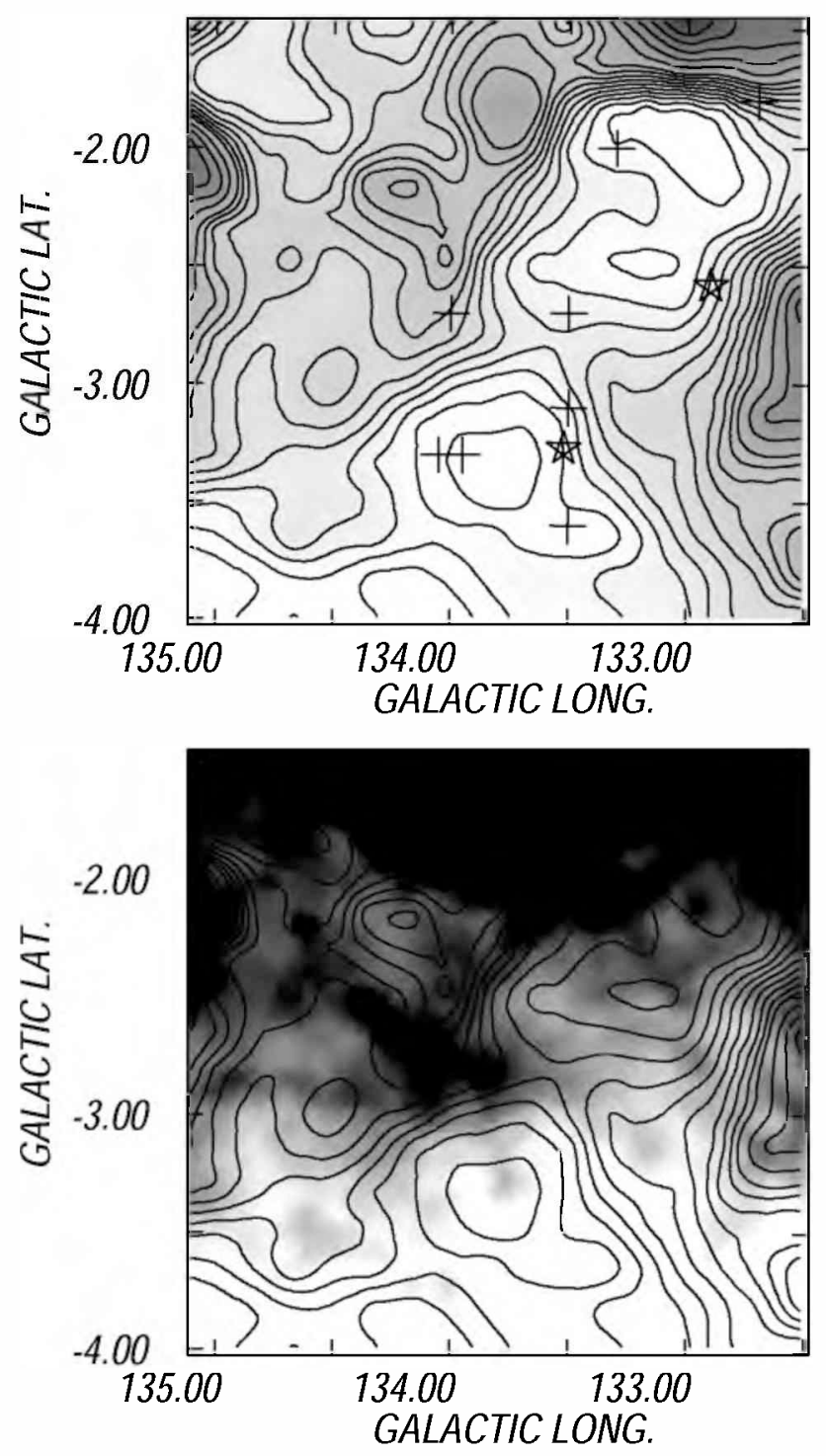

FIG. 7.-Top panel: Latitude-longitude $\mathrm{H}_{\text {I }}$ column density image through the velocity range -24 to $-16 \mathrm{~km} \mathrm{~s}^{-1}$, showing the $\mathrm{H}$ I gas distribution in the vicinity of HD 13022 and HD 13338 . The gray scale corresponds to $(1.8-6.2) \times 10^{20} \mathrm{~cm}^{-2}$. The contour levels are $1.8,2.02 .2$, $2.4,2.6,2.8,3.0,3.2,3.4,3.6,3.8,4.2,4.6,5.0,5.4$, and $5.8 \times 10^{20} \mathrm{~cm}^{-2}$. The stars mark the position of HD 13022 and HD 13338, while the plus signs indicate the position of the other early-type stars (see $\$ 5$ ). Bottom panel: Overlay of the same $\mathrm{H}$ I contour lines and a gray scale $I R A S$ image at 100 $\mu \mathrm{m}$. The gray scale corresponds to $22-35 \mathrm{MJy} \mathrm{sr}^{-1}$.

and HD 13338, indicated as star symbols, appear projected close to the borders of the $\mathrm{H}$ I cavities centered at $(l, b)=$ $(133.15,-2.25)$ and $(l, b)=(133.75,-3: 3)$, respectively. The location of the stars and the kinematic distances derived for the features enable us to associate the $\mathrm{H}$ i structures with the O-type stars.

The structure close to HD 13022 displays two small H I minima. The whole feature is surrounded by neutral clouds toward $l \simeq 133.75$; but due to the location of the structure close to the border of the surveyed region, the presence of an envelope toward $l=132.5$ cannot be confirmed. Consequently, masses and densities were estimated from the mass deficiency in the whole cavity. Three massive stars might also be related to this cavity: HD 13036 (B0.5III), HDE $236954(\mathrm{~B} 3 \mathrm{Ib})$, and $\mathrm{BD}+57^{\circ} 520(\mathrm{~B} 1 \mathrm{II})$.
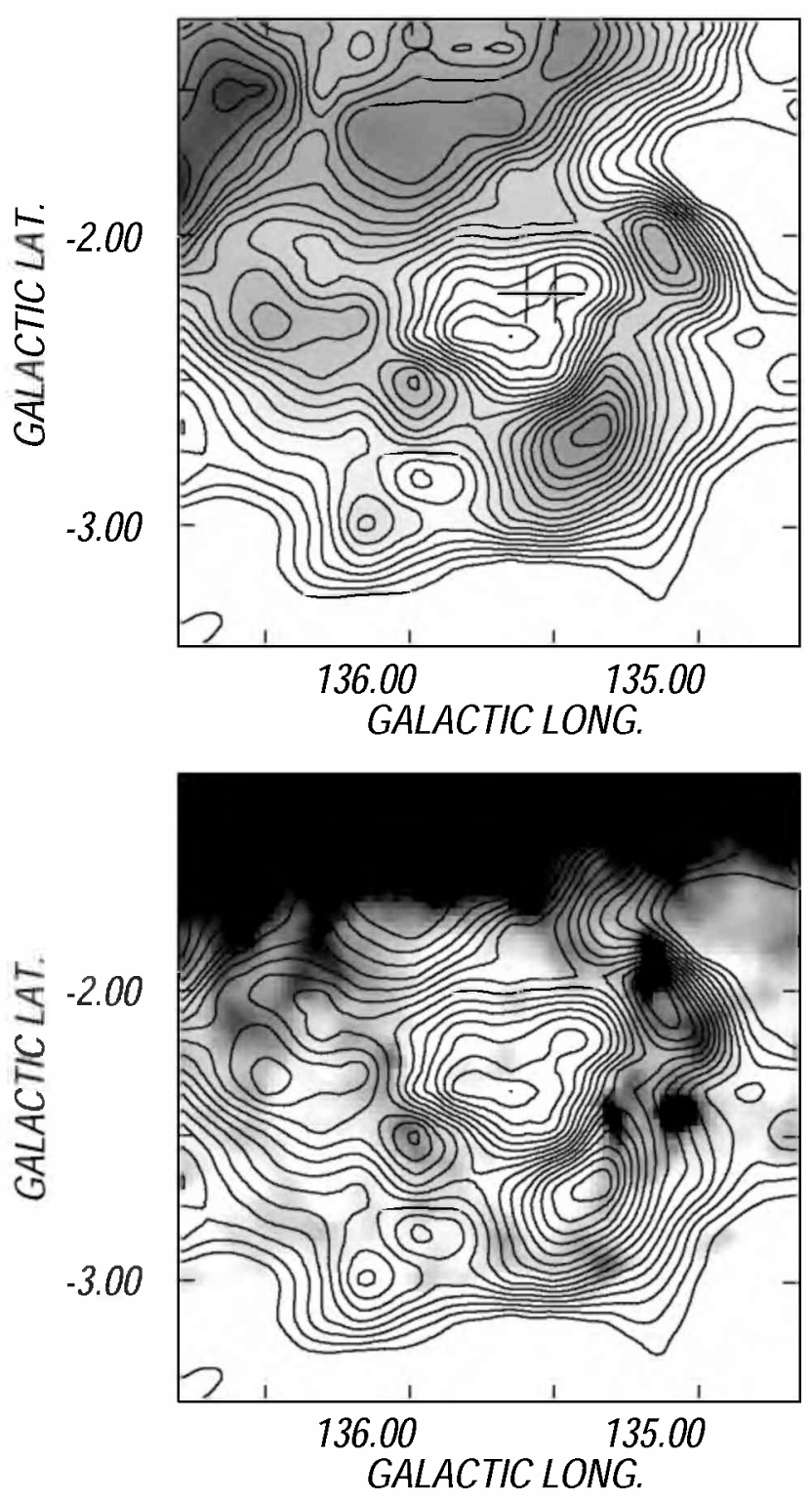

FIG. 8.-Top panel: Latitude-longitude H I column density image through the velocity range -28 to $-18 \mathrm{~km} \mathrm{~s}^{-1}$, showing the $\mathrm{H}$ I gas distribution in the vicinity of HD 15233 and Hilt 233 . The gray scale corresponds to $(5-8.5) \times 10^{20} \mathrm{~cm}^{-2}$. The contour levels are 4.2, 4.4, 4.6, 4.8, $5.0,5.2,5.4,5.6,5.8,6.0,6.2,6.4,6.6,6.8,7.0,7.4,7.8$, and $8.2 \times 10^{20} \mathrm{~cm}^{-2}$. The stars indicate the position of HD 15233 and Hilt 233. Bottom panel: Overlay of the same $\mathrm{H}$ I contour lines and a gray-scale IRAS image at 100 $\mu \mathrm{m}$. The gray scale corresponds to $32-40 \mathrm{MJy} \mathrm{sr}^{-1}$.

The $\mathrm{H}$ I envelope surrounding the void connected to HD 13338 is barely discernible. According to the extension in velocity and systemic velocity, this structure is expanding asymmetrically. Additional early-type stars that can be associated with the structure are HD 13267 (B5Ia), HD 13716 (B0.5III), HD 13758 (B0.5IV), and $\mathrm{BD}+57^{\circ} 513$ (B1III).

The IR emission at $100 \mu \mathrm{m}$ delineates the borders of the structure related to HD 13338 toward larger galactic longitudes and the galactic plane. No clear correlation between $\mathrm{H}$ I and IR emission is apparent for the structure related to HD 13022. Both H I structures are projected onto a region with low radio continuum emission. The cavity closer to the galactic plane appears partially surrounded by enhanced 
radio emission. No $\mathrm{CO}$ emission was detected in connection with these features.

\subsection{The ISM in the Vicinity of the B1III-Type Stars HD 15233 and Hilt 311}

The top panel of Figure 8 shows the distribution of $N_{\mathrm{HI}}$ in the vicinity of HD 15233 and Hilt 311 obtained within the velocity interval -28 to $-16 \mathrm{~km} \mathrm{~s}^{-1}$. The image shows a rather circular cavity centered at $(l, b)=(135.6,-2.2)$, encompassed by a well-defined almost complete shell. The structure presents its largest dimensions and its highest temperature gradient at $-22 \mathrm{~km} \mathrm{~s}^{-1}$, and we adopted this value as the systemic velocity. Since the $\mathrm{H}$ I feature is detected within the velocity interval -32 to $-16 \mathrm{~km} \mathrm{~s}^{-1}$, this systemic velocity suggests the structure is expanding asymmetrically: while the approaching part of the shell expands at $12 \mathrm{~km} \mathrm{~s}^{-1}$, the receding part moves at $8 \mathrm{~km} \mathrm{~s}^{-1}$.

Circular galactic rotation models predict kinematic distances of about $2.2 \mathrm{kpc}$ for this velocity, in close agreement with the distance to Per OB1. Two stars belonging to the association appear located within the cavity: HD 15233 $[(l, b)=(135.6,-2.2)]$ and Hilt $311[(l, b)=(135.5,-2.2)]$ (Garmany \& Stencel 1992), classified as B1III. Their position, close to the center of the void, is marked with crosses in the figure.

As for the other features, the bottom panel of the figure shows the distribution of $N_{\mathrm{H}_{\mathrm{I}}}$ (contour lines) superposed to the IRAS emission at $100 \mu \mathrm{m}$. A clear IR counterpart is seen. The radio continuum map at $2.7 \mathrm{GHz}$ shows a lowemission region that correlates with the $\mathrm{H}$ I void. Based on both morphological and kinematic arguments, the $\mathrm{CO}$ emission detected within the velocity interval -15 to -13.5 $\mathrm{km} \mathrm{s}^{-1}$ may be related to the $\mathrm{H}$ i shell. Thus, the bulk of the observational data discussed here indicate that there is a lack of neutral and ionized gas within the structure.

\section{DISCUSSION}

\subsection{The Origin and Energetics of the Structures}

In order to test if the features surrounding the massive stars can be originated by stellar winds, we have evaluated the mechanical energy $E_{w}$ released into the ISM for the massive stars during the dynamical age $t_{d}$ of the bubbles $\left(E_{w}=L_{w} t_{d}=\dot{M} V_{w}^{2} t_{d} / 2\right)$. The results are summarized in Table 4. The dynamical ages were derived as $t_{d}=$ $0.55 R_{S} / V_{\exp }$, where the constant represents a mean value between the energy and momentum conserving cases. The stellar wind luminosity $L_{w-o}$ and the mechanical energy $E_{w-O}$ were obtained separately for stars HD 14442, HD 14947, HD 16691, HD 13022, HD 13338, and HD 15233 and Hiltner 311. $E_{w-\text { other }}-$ values show the contribution of other massive stars that, according to their location, might contribute in the formation of the structures (indicated as plus signs in the $N_{\mathrm{H}}$ figures). The mass-loss rates for these stars were estimated from the expression by Lamers \& Leitherer (1993), while the terminal velocities correspond to mean values (Prinja et al. 1990). Errors in dynamical ages and mechanical energies are typically $45 \%$ and $70 \%$, respectively.

The fifth and sixth rows of Table 4 show the energy and momentum conversion efficiencies (see Treffers \& $\mathrm{Chu}$ 1982). $\epsilon$ was evaluated as the ratio between the kinetic energy of the neutral structures $E_{k}$ and the mechanical energy provided by all the massive stars in each region $\left(E_{w-o}+E_{w-\text { other }}\right)$; while $\pi$ measures the ratio of the outward momentum of the shell $\left(M_{s} V_{\text {exp }}\right)$ to the integrated momentum carried by the stellar wind of the same stars $\left(\dot{M} V_{w} t_{d}\right)$. Current models for the evolution of interstellar bubbles state $\epsilon \simeq 0.2$ and $\pi \geq 1$ for energy conserving stages and $\epsilon \ll 0.2$ and $\pi=1$ for momentum conserving phases. The uncertainty in $\epsilon$ is about $100 \%$.

Several conclusions can be drawn from the table. The dynamical ages $t_{d}$ are, within errors, roughly compatible with the lifetime in the main sequence of massive stars (Schaerer et al. 1996). $\epsilon \leq 0.01$ for the three structures related to the Of stars HD 14442, HD 14947, and HD 16691. According to evolutionary models of WBBs (e.g., García-Segura \& Mac Low 1995), these values indicate that the stars are capable of blowing the observed interstellar bubbles by means of the stellar wind mechanism. The comparison of $E_{w-O}$ and $E_{w-\text { other }}$ suggests that the three Of stars would be the main contributors to the formation of their own bubbles. Our results also suggest that the bubbles are in the momentum conserving case or in an intermediate stage between energy and momentum conserving cases.

For the bubbles associated with the late O stars HD 13022 and HD 13338, the contribution of other massive stars is important, being half the mechanical energy input in the case of HD 13022. The $\epsilon$-values are in the range 0.160.02. Again in this case, the hypothesis of a stellar wind origin may be held. Within errors, these bubbles appear to be closer to the energy conserving case than the bubbles related to the Of stars.

The $\epsilon$-value derived for the $\mathrm{H}$ I structure related to the two B stars, clearly indicates that this feature has been originated by a different mechanism since a stellar wind origin is difficult to support. Similar H I structures connected to supernova remnants have also been found; and a supernova explosion in Per OB1 cannot be ruled out. The origin of this $\mathrm{H}$ I feature will be analyzed elsewhere.

TABLE 4

Derived Parameters

\begin{tabular}{|c|c|c|c|c|c|c|}
\hline Star & HD 14442 & HD 14947 & HD 16691 & HD13022 & HD13338 & $\begin{array}{c}\text { HD15233 } \\
\text { Hilt } 311\end{array}$ \\
\hline$L_{w-o}\left(10^{36} \operatorname{ergs~s}^{-1}\right) \ldots \ldots$ & 5.2 & $3.5-33$ & $12-31$ & 0.3 & 0.07 & 0.015 \\
\hline$t_{d}\left(10^{6} \mathrm{yr}\right) \ldots \ldots \ldots \ldots \ldots$ & 2.0 & 1.0 & 1.8 & 1.0 & 1.4 & 1.0 \\
\hline$E_{w-o}\left(10^{50} \mathrm{ergs}\right) \ldots \ldots \ldots$ & 3.2 & $1-10$ & $7-17$ & 0.09 & 0.03 & 0.006 \\
\hline$E_{w-\text { other }}\left(10^{50}\right.$ ergs $) \ldots \ldots \ldots$ & 0.12 & & 0.17 & 0.045 & 0.12 & $\ldots$ \\
\hline 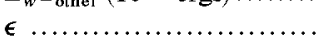 & 0.006 & $0.01-0.001$ & $0.001-0.0004$ & $0.2-0.1$ & $0.1-0.02$ & 1.6 \\
\hline$\pi \ldots \ldots \ldots \ldots \ldots \ldots \ldots \ldots$ & 0.6 & $0.3-1.8$ & $0.1-0.3$ & 14 & 4 & 90 \\
\hline$C_{\mathrm{ax}} \ldots \ldots \ldots \ldots \ldots \ldots \ldots$ & 1.7 & 2.0 & 1.7 & 1.5 & 1.0 & $\ldots$ \\
\hline$C_{\text {off }} \ldots \ldots \ldots \ldots \ldots \ldots \ldots \ldots \ldots \ldots$ & 0.5 & 0.7 & 0.6 & 0.6 & 0.3 & $\ldots$ \\
\hline
\end{tabular}


The low $\epsilon$-values derived for the new $\mathrm{H}$ I interstellar bubbles could also be explained if a substantial amount of the energy provided by the stars has released into the large $\mathrm{H}$ I structure related to Per OB1 as a whole. Although this leakage cannot be ruled out, we note that similar low values were found for most of the interstellar bubbles associated with Wolf-Rayet and Of stars (e.g., Treffers \& Chu 1982; Wrigge 1999; Benaglia \& Cappa 1999; Cappa et al. 1999, and references therein). As pointed out by García-Segura \& Mac Low (1995) and Wrigge (1999) an overestimation of the stellar wind parameters for massive stars cannot be ruled out.

Finally, it is of interest to evaluate the energetics of the large $\mathrm{H}$ I shell related to Per OB1 as a whole. The kinetic energy $E_{k}$ of the shell is $\approx 9 \times 10^{49}$ ergs. Taking into account that for an energy conserving bubble $E_{k}=0.2$ $E_{w-\min }, E_{w-\min }$ results $\simeq 4.5 \times 10^{50} . E_{w-\min }$ represents the minimum stellar wind mechanical energy necessary to blow the observed $\mathrm{H}$ I shell. At the same time, we estimated the energy $E_{w}$ released into the ISM by all the B stars in the association through the stellar wind mechanism. Here, we take into account only the $\mathrm{B}$ stars since some of the $\mathrm{O}$ and Of stars have blown their own bubbles and the contribution of these stars to the energetics of the large $\mathrm{H}$ I shell is difficult to evaluate. Consequently, we believe that the derived $E_{w}$-value represents a lower limit to the stellar wind energy available to blow the large $\mathrm{H}$ I shell. $\dot{M}$ and $V_{w}$ for the B stars were obtained using the fitting formula by Lamers \& Leitherer (1993) and mean values by Prinja et al. (1990), respectively. Adopting the age of the shell to be $7 \times 10^{6}$ yr (see $\S 1$ ), $E_{w}$ turns out to be about $1.3 \times 10^{51}$ ergs. Then $E_{k} / E_{w} \simeq 0.07$, indicating that Per OB1 suffices to blow the large $\mathrm{H}$ i shell.

\subsection{Morphological Characteristics}

We evaluated two parameters to characterize the morphology of the $\mathrm{H}_{\mathrm{I}}$ interstellar bubbles: the ratio of the major to the minor axis of the cavity $C_{\mathrm{ax}}=2 r_{M} / 2 r_{m}$; and $C_{\text {off }}$, which is the ratio of the observed projected distance of the star responsible for creating the bubble from its center to the minor axis of the cavity (Arnal 1992). These parameters enable us to quantify the ellipticity of the $\mathrm{H}$ I interstellar bubbles and the eccentric location of the stars.

The values for $C_{\mathrm{ax}}$ and $C_{\mathrm{off}}$ are listed in Table 4. $C_{\mathrm{ax}}$ varies between 1.0 and 2.0, while $C_{\text {off }}$ ranges from 0.3 to 0.7 . Both parameters were also evaluated for the $\mathrm{H}$ I bubbles surrounding the Of stars HD 91572 and HD 91824, HD 94963, HD 112244, HD 152386, HD 155913, and HD 175758 and HD 175876 (Cappa \& Benaglia 1998; Benaglia \& Cappa 1999). $C_{\mathrm{ax}}-$ values are within the range 1.0 to 1.4 , while $C_{\text {off }}$ varies between 0.1 and 0.7 . Similar values can also be obtained for $\mathbf{H}$ I bubbles related to WR stars (e.g., Niemela \& Cappa de Nicolau 1991; Arnal 1992; Cappa et al. 1996a, 1996b; Arnal \& Cappa 1996; Arnal \& Roger 1997; Gervais \& St-Louis 1999).

Most of the $\mathbf{H}$ I bubbles surrounding WR and Of stars are elliptical rather than spherical and most of the stars are eccentric with respect to the center of the bubbles. In addition, many stars are located closer to one of the highest density borders of the cavities. It is worth mentioning that the eccentric location is also seen in optical ring nebulae (e.g., Miller \& Chu 1993; Marston et al. 1994).

Aspherical interstellar bubbles can be explained by relaxing some of the basic assumptions adopted in the classical evolutionary models of interstellar bubbles (e.g., McCray 1983). An inhomogeneous ISM in the environs of the star and/or a star moving with a large spatial velocity with respect to its local ISM can easily favor the formation of distorted bubbles (e.g., Weaver et al. 1977; Dyson 1977; Icke 1988; Brighenti \& D'Ercole 1995).

The spatial velocities of the stars can be derived from radial velocities and proper motions. The stellar radial velocities (see Table 1) are, in all cases, more negative than the systemic velocities of the associated $\mathrm{H}$ I bubbles. As pointed out by Conti, Leep, \& Lorre (1977) and Gies (1987), Of stars show systematically negative velocities due to the atmospheric outflow, and consequently, their radial velocities are not reliable measures of their velocities in space. The radial velocities observed in direction to other O-type stars in Per OB1 are also more negative than what is expected from circular galactic rotation models (see Table 1). HD 12993 and HD 13268 are runaway stars (Cruz-González et al. 1974; McLachlan \& Nandy 1985).

Proper motions for the $\mathrm{O}$ and $\mathrm{O}$ stars related to the individual bubbles were looked for in the Tycho-2 Catalog (Hog et al. 2000). The components of the proper motions for these stars are below $2 \sigma$ except for HD 16691, for which the components are $\leq 3 \sigma$. In spite of the large uncertainties, the derived tangential velocities suggest that HD 16691 is moving away from the galactic plane. Taking into account the location of the star, this fact casts doubts on the $\mathrm{H} \mathrm{I}$ structure-star association.

Taking these facts into account, an inhomogeneous ISM seems to be the most probable reason for the eccentric position of the star and the aspherical appearance of the probable $\mathrm{H}$ I bubbles in Per OB1.

Concerning the other O-type stars in the association, HD 12993, HD 14434 and Hilt 23 are close to the borders of the region surveyed with the Effelsberg radio telescope, and we were not able to examine their neighborhood with these data; while HDE 236894, HD 12323, HD 13268, HD13745, and HD 15642 are outside the surveyed region.

No $\mathrm{H}$ I bubble candidate was found to be related to HD 237019 within the velocity interval -50 to $-15 \mathrm{~km} \mathrm{~s}^{-1}$. Several reasons may be invoked in order to explain this fact. If present, the $\mathrm{H}$ I structure related to this star could be smaller than the HPBW of the Effelsberg radio telescope at $21 \mathrm{~cm}$, i.e., $\simeq 5 \mathrm{pc}$ at $2.3 \mathrm{kpc}$. A high ambient gas density might be responsible for the small size of such bubble. On the other hand, confusion effects originated in the presence of fore- and background $\mathrm{H}$ I gas with velocities in the range -30 to $-18 \mathrm{~km} \mathrm{~s}^{-1}$ might also mask an $\mathrm{H}$ i bubble related to this star.

\section{SUMMARY}

Based on $H_{\text {I }} 21 \mathrm{~cm}$ line observations, we have investigated the ISM in the vicinity of the $\mathrm{O}$ and $\mathrm{O}$ stars belonging to the Per OB1 association, searching for the signatures of the interaction between the stellar wind and the surrounding gas.

The Effelsberg $\mathrm{H}$ I data $(\mathrm{HPBW}=8.4)$ allowed us to discover probable interstellar wind-blown bubbles associated with the massive Of stars HD 14442 [O5n(f)p] and HD $14947[\mathrm{O} 5 \mathrm{If}+]$ and with the O stars HD 13022 [O9.5II$\mathrm{III}((\mathrm{n}))]$ and HD 13338 [O9.5V], while there are some doubts on the detection of an $\mathrm{H}$ I bubble related to HD 16691 [O5If +$]$. The dimensions of these interstellar bubbles are in the range $18-33 \mathrm{pc}$ and their expansion 
velocities are of about $10 \mathrm{~km} \mathrm{~s}^{-1}$, implying dynamical ages of about (1-2) $\times 10^{6}$ yr. Infrared counterparts were also found for these bubbles. Our results indicate that the $\mathrm{O}$ and Of stars would be the main contributors to the shaping of the bubbles through stellar wind-ISM interaction. The H I bubbles discovered in Per OB1 share some characteristics with previously found $\mathrm{H}$ I interstellar bubbles related to WR and Of stars: (1) these new bubbles would be in the momentum-conserving case or in an intermediate stage between energy and momentum-conserving cases, (2) they are elliptical in shape, and (3) the $\mathrm{O}$ and $\mathrm{O}$ stars responsible for their formation are projected at a eccentric position with respect to the center of the bubble.

We have also found a clear $\mathrm{H}$ I shell-like feature that seems to be related to the B1III stars HD 15233 and Hilt 311. Based on the present results, this feature cannot be explained as a wind-blown bubble. It must have been originated by a different mechanism.

The large-scale $\mathrm{H}$ I maps obtained with lower angular resolution data $\left(\mathrm{HPBW}=36^{\prime}\right)$ enabled the identification of an $\mathrm{H}$ I shell that encircles the OB association Per OB1. The shell, which is $350 \times 550 \mathrm{pc}$ in size, could have been blown by Per OB1 as a whole. An IR counterpart of this shell was also found.

We are very grateful to the anonymous referee, whose comments and suggestions led to the improvement of this paper. C. E. C. is grateful to V. S. Niemela for suggesting Per OB1 for this study and to the computational staff of the IAR. This research was partially supported by CONICET project PIP 607/98.
Arnal, E. M. 1992, A\&A, 254, 305

Arnal, E. M., \& Cappa, C. E. 1996, MNRAS, 279, 788

Arnal, E. M., Cappa, C. E., Rizzo, J. R., \& Cichowolski, S. 1999, AJ, 118, 1798

Arnal, E. M., \& Roger, R. 1997, MNRAS, 285, 253

Becker, W., \& Fenkart, R. 1971, A\&AS, 4, 241

Benaglia, P., \& Cappa. C. 1999, A\&A, 346, 979

Brand, J., \& Blitz, L. 1993, A\&A, 275, 67

Brighenti, F., \& D'Ercole, A. 1995, MNRAS, 273, 443

Bruhweiler, F. C., Gull, T. R., Kafatos, M., \& Sofia, S. 1980, ApJ, 238, L27

Burton, W. B. 1988, In Galactic and Extragalactic Radio Astronomy, ed. G. L. Verschuur \& K. I. Kellerman (2d ed.; Berlin: Springer), 295

Cappa, C. E., \& Benaglia, P. 1998, AJ, 116, 1906

Cappa, C. E., Dubner, G. M., Rogers, C., \& St-Louis, N. 1996a, AJ, 112, 1104

Cappa, C., Goss, W. M., Niemela, V., \& Ostrov, P. 1999, AJ, 118, 948

Cappa, C. E., Niemela, V. S., Herbstmeier, U., \& Koribalski, B. 1996b, A\&A $, 312,283$

Cappa de Nicolau, C. E., \& Olano, C. A. 1990, Rev. Mex. Astron. Astrofis., 21,273

Caswell, J. L. \& Haynes, R. F. 1987, A\&A, 171, 261

Chlebowski, T., \& Garmany, C. D. 1991, ApJ, 368, 241

Chromey, F. R., Elmegreen, B. G., \& Elmegreen, D. M. 1989, AJ, 98, 2303

Conti, P. S., Leep, E. M., \& Lorre, J. J. 1977, ApJ, 214, 759

Cruz-González, C., Recillas-Cruz, E., Costero, R., Peimbert, M., \& Torres-

Peimbert, S. 1974, Rev. Mex. Astron. Astrofis., 1, 211

de Geus, E. J. 1988, Ph.D. thesis, Univ. Leiden

Dyson, J. E. 1977, A\&A, 59, 161

Fürst, E., Reich, W., Reich, P., \& Reif, K. 1990, A\&AS, 85, 691

García-Segura, G., \& Mac Low, M.-M. 1995, ApJ, 455, 145

Garmany, C. D., \& Stencel, R. E. 1992, A\&AS, 94, 211

Gervais, S., \& St-Louis, N. 1999, AJ, 118, 2394

Gies, D. R. 1987, ApJS, 64, 545

Groenewegen, M. A. T., \& Lamers, H. J. G. L. M. 1989, A\&AS, 79, 359

Hartman, D., \& Burton, W. B. 1997, Atlas of Galactic Neutral Hydrogen (Cambridge: Cambridge Univ. Press)

Heiles, C. 1979, ApJ, 229, 533 1984, ApJS, 55, 585

Heyer, M. H., Brunt, C., Snell, R. L., Howe, J. E., Schloerb, F. P., \& Carpenter, J. M. 1998, ApJS, 115, 241

Hog, E., Fabricius, C., Makarov, V. V., Urban, S., Corbin, T., Wycoff, G., Bastian, U., Schwekendiek, P., \& Wicenec, A. 2000, A\&A, 355, L27

Howarth, I. D., Siebert, K. W., Hussain, G. A. J., Prinja, R. K. 1997, MNRÁS, 284, 265

Humphreys, R. 1978, ApJS, 38, 309

Icke, V. 1988, A\&A, 202, 177

Janes, K., \& Adler, D. 1982, ApJS, 49, 425

Kalberla, P. M. W., Mebold, U., \& Reich, W. 1980, A\&A, 82, 275

Kalberla, P. M. W., Mebold, U., \& Reif, K. 1982, A\&A, 106, 190

\section{REFERENCES}

Kendall, T. R., Lennon, D. J., Brown, P. J. F., \& Dufton, P. L. 1995, A\&A, 298,489

Kimeswenger, S., \& Weinberger, R. 1989, A\&A, 209, 51

Koo, B.-C., Heiles, C. \& Reach, W. T. 1992, ApJ, 390, 108

Kuchar, T. A., \& Bania, T. M. 1993, ApJ, 414, 664

Lamers, H. J. G. L. M., \& Leitherer, C. 1993, ApJ, 412, 771

Leitherer, C. 1988, ApJ, 326, 356

Lockman, F. J. 1989, ApJS, 71, 469

Lozinskaya, T. A. 1982 , Ap\&SS, 87, 313

Mac Low, M.-M., \& McCray, R. 1988, ApJ, 324, 776

Maeder, A., \& Meynet, G. 1994, A\&A, 287, 803

Marston, A. P. 1996, AJ, 112, 2828

Marston, A. P., Yocum, D. R., García-Segura, G., \& Chu, Y.-H. 1994, ApJS, 95, 151

Mathys, G. 1989, A\&AS, 81, 237

McCray, R. 1983, Highlights Astron., 5, 567

McCray, R., \& Kafatos, M. 1987, ApJ, 317, 190

McLachlan, A. \& Nandy, K. 1985, MNRAS, 215, 473

Miller, G., \& Chu, Y.-H. 1993, ApJS, 85, 137

Münch, G. 1957, ApJ, 125, 42

Nicolet, B. 1978, A\&AS, 34, 1

Niemela, V. S., \& Cappa de Nicolau, C. E. 1991, AJ, 101, 572

Normandeau, M., Taylor, A. R., \& Dewdney, P. E. 1996, Nature, 380, 687

Oey, M. S. 1996, ÄJ, 467, 666

Parker, R. A. R., Gull, T. R., \& Kirschner, R. P. 1979, An Emission Line Survey of the Milky Way, NASA SP No. 434

Phillips, A. P. \& Gondhalekar, P. M. 1981, MNRAS, 196, 533

Prinja, R. K., Barlow, M. J., \& Howarth, I. D. 1990, ApJ, 361, 607

Puls, J., et al. 1996, A\&A, 305, 171

Reich, W., Reich, P., \& Fürst, E. 1990, A\&AS, 83, 539

Rizzo, J. R. 1994, Ph.D. thesis, Univ. Nac. La Plata

Rizzo, J. R., \& Bajaja, E. 1994, A\&A, 289, 922

Ruprecht, J. 1966, Trans. IAU, 12B, 350

Sancici, R. 1974, in IAU Symp. 60, Galactic Radio Astronomy, ed. F. J. Kerr \& S. C. Simonson, III (Dordrecht : Reidel), 115

Schaerer, D., de Koter, A., Schmutz, W., \&Maeder, A. 1996, A\&A, 310, 837

Schmidt-Kaler, Th. 1982, in Landolt-Börnstein, Numerical Data and Functional Relationships in Science and Technology, ed. K. Schaifers \& H. H. Voigt, Vol. VI/2b (New York: Springer), 1

Scuderi, S., Panagia, N., Stanghellini, C., Trigilio, C., \& Umana, G. 1998, A\&A, 332, 251

Simonson, S. C., \& van Someren Greve, H. W. 1976, A\&A, 49, 343

Treffers, R. R., \& Chu, Y.-H. 1982, ApJ, 254, 569

Underhill, A. B., \& Gilroy, K. K. 1990, ApJ, 364, 626

Vacca, W. D., Garmany, C. D., \& Schull, J. M. 1996, ApJ, 460, 914

Walborn, N. 1972, AJ, 77, 312

. 1973, AJ, 78, 1067

Weaver, R., McCray, R., Castor, J., Shapiro, P., \& Moore, R. 1977, ApJ, 218,377

Wrigge, M. 1999, A\&A, 343, 599 